\title{
DEVELOPMENT OF WEARABLE HUMAN FALL DETECTION SYSTEM USING MULTILAYER PERCEPTRON NEURAL NETWORK
}

\author{
HAMIDEH KERDEGARI \\ Department of Computer and Communication Systems Engineering, University Putra Malaysia \\ 43300 Serdang, Selangor, Malaysia \\ E-mail: hamideh.kerdegari@gmail.com \\ www.upm.edu.my \\ KHAIRULMIZAM SAMSUDIN \\ Department of Computer and Communication Systems Engineering, University Putra Malaysia \\ 43300 Serdang, Selangor, Malaysia \\ E-mail:kmbs@eng.upm.edu.my \\ www.upm.edu.my \\ ABDUL RAHMAN RAMLI \\ Department of Computer and Communication Systems Engineering, University Putra Malaysia \\ 43300 Serdang, Selangor, Malaysia \\ E-mail: arr@eng.upm.edu.my \\ www.upm.edu.my \\ SAEID MOKARAM \\ Department of Computer and Communication Systems Engineering, University Putra Malaysia \\ 43300 Serdang, Selangor, Malaysia \\ E-mail: saeed.mokaram@gmail.com \\ www.upm.edu.my \\ Received 2 August 2012 \\ Accepted 4 september 2012
}

\begin{abstract}
This paper presents an accurate wearable fall detection system which can identify the occurrence of falls among elderly population. A waist worn tri-axial accelerometer was used to capture the movement signals of human body. A set of laboratory-based falls and activities of daily living (ADL) were performed by volunteers with different physical characteristics. The collected acceleration patterns were classified precisely to fall and ADL using multilayer perceptron (MLP) neural network. This work was resulted to a high accuracy wearable fall-detection system with the accuracy of $91.6 \%$.
\end{abstract}

Keywords: Wearable fall detection system, Tri-axial accelerometer, Classification, Multilayer perceptron

\section{Introduction}

As we grow old, our desire for being independent does not decrease while our health needs to be monitored more frequently. Accidents such as falling can be a serious problem for the elderly. Increasing worldwide elderly population could augment serious effects and problems caused by fall. Therefore, falls have been recognized as a major health problem in the aging population. More seriously, falls cause more than $90 \%$ of all hip fractures and $20 \%$ die within a year of the 
fracture. ${ }^{1}$ Falls affect the quality of life among elderly people by producing fear, decreasing in independency and resulting in a decline in mobility and activity. A serious consequence of falling is the "long-lie", defined as remaining on the ground or floor for more than an hour following a fall. The long-lie is associated with high mortality rates among the elderly. A fall detection system can reduce the occurrence of the "long-lie" by minimizing the time between the fall and the arrival of medical help. ${ }^{2}$ Nowadays, human fall detection has been an active research topic due to that the healthcare industry has a big demand for products and technology of fall detection. ${ }^{3}$ Since there are many serious effects caused by falls, an efficient system that is designed to detect and prevent falls will benefit the elderly society. In this paper, an intelligent wearable fall detection system has been proposed. Wearable fall detection systems usually use different sensors such as accelerometers, gyroscopes and in some cases both of them to distinguish falls from ADL. The collected data by wearable sensors can be processed by a remote PC or by an embedded system using classification algorithms. As wearable devices are standalone devices, the elderly people with a wearable device are independent anywhere and still be constantly monitored.

The contributions in this paper are summarized as follows:

- A wearable fall detection system was proposed which has composed of a tri-axial accelerometer (ADXL345) for capturing different human movements signals while attached to the volunteer's waist. This wearable device can be used in both indoor and outdoor environment and because of its lightweight does not make any disturbance for elderly people.

- A complete experiment log of different types of movements was proposed which fall and ADL in all directions were considered in order to cover different types of fall that may happen and cause injury to an elderly person.

- Windowing technique and feature extraction were used in order to increase the classification accuracy before raw acceleration data going through classification algorithm.

- MLP neural network was applied as a classification algorithm for precise classification of the collected falls and ADL acceleration patterns to a reasonable degree of accuracy. Results showed that MLP neural network can detect falls with relatively high accuracy compared with other existing methods.

\section{Related Works}

Fall detection systems can be classified into three approaches: wearable device, video-based device and ambience device. Wearable device approaches are classified as one of the most preferred and relatively applicable type of fall detection methods. The wearable device approach is to hold or wear some devices with embedded sensors to detect the posture and motion of the wearer's body and use classifiers to identify fall movements from ADL movements. ${ }^{3}$ Video-based approach uses camera as a vision sensor which cameras detect multiple events simultaneously and then the recorded video can be used for remote and post verification and analysis. The disadvantage of this approach is its very limited range of operation. Due to the fixed nature of the camera, it can only be used in indoor environments. The infrastructure required for such approach is expensive for reliable detection as a camera needs to be installed in every room and other places in the living environment. Connecting the cameras to a single dedicated server also requires communication link to be established. All these factors contribute to increasing the cost involved for deploying such a system. Other limitations include camera blind spots and occlusions due to the presence of a dynamic environment. ${ }^{3,4}$ Ambience device approach is to use multiple installed sensors to collect the data from a person when he/she is close to them. ${ }^{3}$ The drawback of this approach is that these systems are only suitable for indoor environments. They are not well suited for a normal living environment because the excessive noise in a normal living environment decreases the signal obtained from the sensors. ${ }^{4}$ When compared with videobased and ambience device approach, wearable device approach can easily be used in normal living environment for fall detection among elderly people. The most important advantage is that an elderly person with a wearable device can go in an outdoor environment and still be monitored. This method does not suffer from noise problems and living environment limitations. Kangas et $\mathrm{al}^{5}$ used body attached triaxial accelerometer to detect falls. The acceleration signal was measured with body attached accelerometers from intentional falls and ADL to determine threshold values for multiple parameters capable of discriminating 
between falls and ADL. Therefore, a fall is considered detected as long as one threshold is exceeded. Bourke et $\mathrm{al}^{6}$ used a tri-axial accelerometer to detect fall and ADL. This was accomplished using a single threshold determined by the fall-event data-set, applied to the resultant-magnitude acceleration signal from a tri-axial accelerometer located at the trunk. Using the thresholdbased algorithm is not an optimal method for fall detection because the value ranges overlap for different parameters measured using accelerometer for different types of fall and ADL. Zheng et $\mathrm{al}^{7}$ described a fall detection system using a 3-axis accelerometer and a wireless alert system. The device is worn on the waist using a two-stage fall detection algorithm, which senses rapid impact and body orientation of the wearer. Since all simulated activities except for ADL were performed on a large soft mat, it is doubtable that whether the detecting thresholds adapt to the actual falls on the solid ground. Bourke et $\mathrm{al}^{2}$ developed a fall detection system using a tri-axial accelerometer, microcontroller, battery and Bluetooth module. This sensor is attached to a custom designed vest, designed to be worn by the elderly person under clothing. The fall detection algorithm was developed and incorporates both impact and posture detection capability. The limitation of this method is that only young volunteers performed the simulated activities for the reason that the experiment is dangerous to the elderly people and however further long-term testing using elderly subjects is required to validate the systems performance. Zhang et $\mathrm{al}^{8}$ used a wearable tri-axial accelerometer to monitor the human movement, and proposed a novel fall detection algorithm based on non-negative matrix factorization (NMF). The K-NN approach was used to determine whether a fall occurred. However, the database did not cover different types of fall and ADL in order to best simulate the type of fall that may happen and cause injury to the elderly. Zhang et $\mathrm{al}^{9}$ used a wearable triaxial accelerometer to capture the movement data of the human body, and proposed a fall detection method based on one-class SVM. The drawback of this method relates to the algorithm which is the complexity in calculation, especially for the pre-processing.

\section{Methodology}

This section introduces the general scheme of the proposed wearable fall detection system. First, accelerometer sensor as a wearable device and its placement on human's body will be explained. Subsequently, data collection using tri-axial accelerometer and the process of data labeling and data trimming will be described. Windowing technique is used to divide the sensor signal into smaller time segments and related features from each window will be extracted. Finally, MLP neural network is applied for precise classification of motions and determination of fall or ADL events.

\subsection{Accelerometer sensor}

Detection of fall using a wearable device commonly employs various sensors such as accelerometer and gyroscope. Acceleration is the main characteristic of fall and accelerometer-based method has been popular with wearable device due to its low-cost, convenience and high accuracy. On the other hand, gyroscopes are rarely found in wearable device due to relatively high-cost. Therefore, using an accelerometer is more practical. In this work, fall and ADL acceleration signals were measured using a mounted tri-axial accelerometer on the volunteer's body.

\subsubsection{Tri-axial accelerometer (ADXL345)}

The ADXL345 is a small, thin and low power 3-axis accelerometer. It measures accelerations up to $+/-16 \mathrm{~g}$ with high resolution (13-bit) and the static acceleration of gravity in tilt-sensing applications, as well as dynamic acceleration resulting from motion or shock. Its high resolution $(4 \mathrm{mg} / \mathrm{LSB})$ enables the measurement of inclination changes less than $1.0^{\circ}$. Several special sensing functions are also embedded in ADXL345 like activity and inactivity sensing, tap sensing and free-fall sensing ${ }^{10}$. The ADXL345 Evaluation Board as shown in Fig. 1 is a simple solution for working with the ADXL345 triple axis digital accelerometer. The evaluation board includes an ADXL345, SD card socket

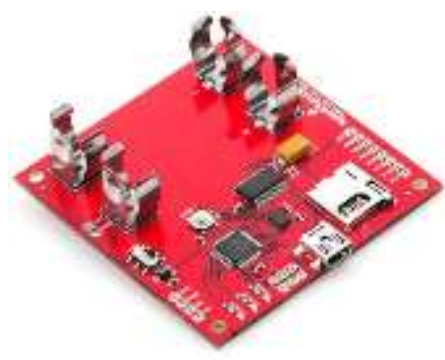

Fig. 1. ADXL345 evaluation board 
for data logging, a USB port for serial communication with a computer and an Atmega328 with Arduino Bootloader $^{11}$. Using Arduino Serial Bootloader (Atmega328) means that the device can be reprogrammed via the USB connector using the Arduino IDE, as well as by standard programming methods using the 6 pin ISP header. The device, which is powered by two AA batteries, allows the user to manipulate the register settings of the ADXL345 using a serial command interface. All of the data which is read from the accelerometer can be logged to the microSD card via a FAT32 file system. It enables the evaluation board to work and log acceleration data in a standalone manner. When the device is powered on and if a card is found, a new log file will be created on MicroSD card. The log files are created with file names in the format 'ADXL345_XXX.csv.' The XXX is a 3-digit number that increments every time a new file is created. In this work, the sensor was initialized to $\log$ data at $100 \mathrm{~Hz}$ with $+/-2 \mathrm{~g}$ range to record high resolution acceleration signals. Since acceleration Hex values are recorded in ASCII format $\log$ files, a Matlab function was implemented to read and parse 3-axis data and return them in decimal numeric format for further processing.

\subsubsection{Sensor placement}

Recognition of the most appropriate position of the sensor on the wearers' body is important in order to receive clear signals of their movement. Several works have demonstrated experiments on attaching acceleration sensors at different parts of the body like the waist, wrist, chest, head and thigh. $5,7,12$ and 13 The waist has been suggested to be the most suitable, since at this location the acceleration signal is similar and evenly distributed between different fall types. Furthermore, waist-attached accelerometers are located near to the body center of gravity providing reliable information on the subject's movements. ${ }^{5}$ In this work, all the volunteers were asked to fasten the acceleration sensor with the provided belt to their waists. Fig. 2 shows the exact location of ADXL345 accelerometer sensor with its 3-axis direction while fastened on the volunteer's waist.

\subsection{Data collection}

In this work, a complete database of fall and ADL patterns was collected by some experiments. These experiments were carried out by 50 volunteers. The

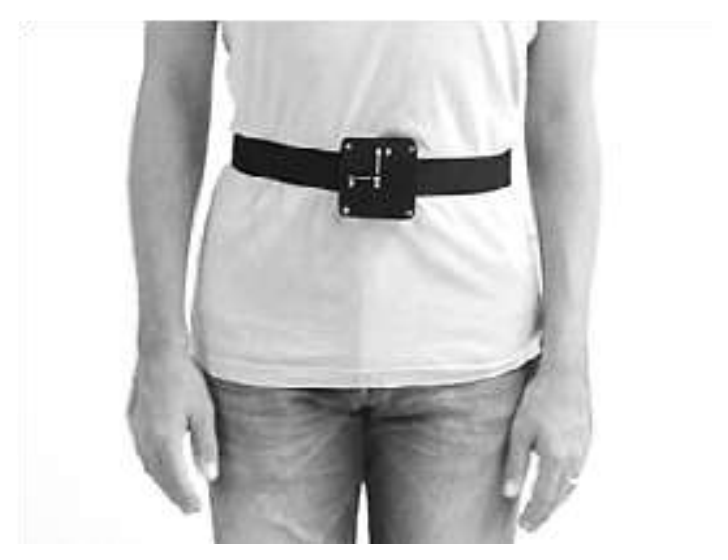

Fig. 2. Sensor placement on volunteer's body

volunteers were 18 males and 32 females with the average age, height and weight of 32 years old, $165 \mathrm{~cm}$ and $66 \mathrm{~kg}$ respectively. In order to collect the acceleration data, each volunteer was asked to perform 9 different ADL activities and 11 different fall activities inside the laboratory with the sensor attached to their waist. Each activity was performed on a large mattress and each volunteer was advised to perform the experiment as naturally as possible. However, since these experiments can be dangerous for the elderly, the database was made mostly by younger volunteers and just one volunteer considered was 65 years old. In total, 1000 recorded movement acceleration data have been collected. Table 1 is the experiment log that has been completed for each volunteer. Fall and ADL in all directions were considered in order to cover different

Table 1. Experiment log including personal information, ADL and fall patterns.

\begin{tabular}{lll}
\hline $\begin{array}{l}\text { Personal } \\
\text { information }\end{array}$ & ADL patterns & Fall patterns \\
\hline Name & Walking & Forward \\
Sex & Running & Backward \\
Age & Jumping & Backward \\
Weight & Sitting on chair & Backward base on wall \\
Height & Sitting on ground & Side left with flexed knee \\
& Sitting to standing (chair) & Side left base on wall \\
& Sitting to standing (ground) & Backward and turn to left \\
& Standing to lying position & Side right with flexed knee \\
& Lying to standing position & Side right base on wall \\
& & Backward and turn to right \\
& & Fall as you like \\
\hline
\end{tabular}


types of fall that may happen and cause injury to an elderly person. $^{14}$

Fig. 3 shows different types of ADL and fall experiments which were performed by volunteers respectively. The total sum vector of acceleration in a sample walking and fall forward pattern have been shown in Fig. 4. A big change in acceleration signal during the first two seconds of data collection shows the start point of volunteers' movement which has done with a tap on the sensor by volunteers. In Fig. 4, part (b) after some walking there is a big change in acceleration signal which shows the occurrence of fall. It is obvious that at the start of falling, the earth gravity acceleration decreases from one $G$ to near zero which shows a free fall.

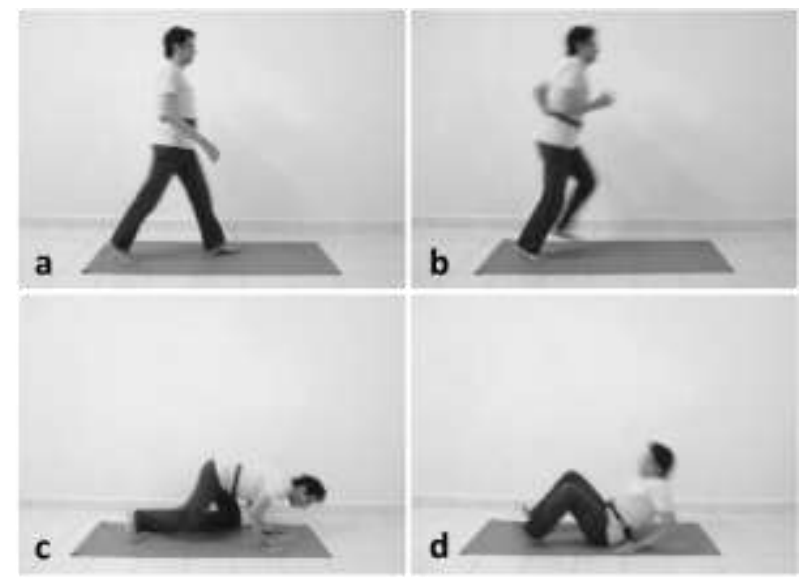

Fig. 3. Different types of ADL and fall experiments a. Walking, b. Running, c. Fall forward, d. Fall backward
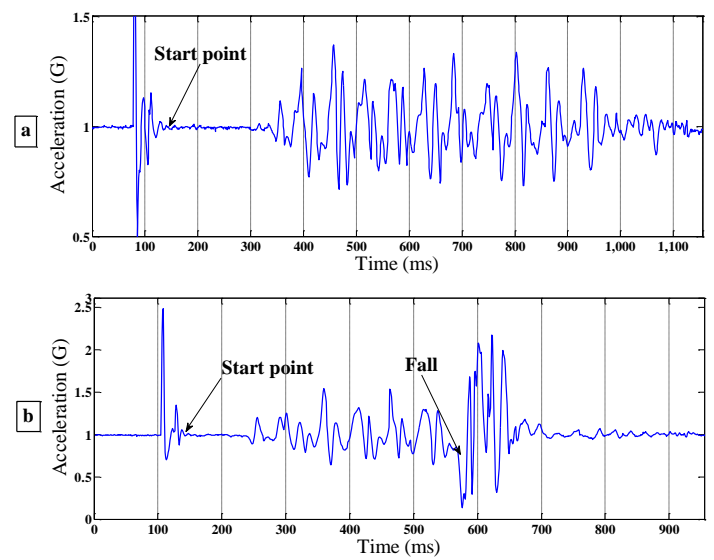

Fig. 4. a. Sample of walking acceleration signal, b. Sample of fall forward acceleration signal

\subsubsection{Data trimming and data labeling}

In order to find the start moment of recording data in the experiments, all the volunteers were asked to knap on the ADXL345 evaluation board whenever they launched to perform the experiments. This knapping can be observed in Fig. 4 during the first two seconds of data collection which is a big change in acceleration data. The start and end of each collected sample data was trimmed to remove all the irrelevant captured data that may happen during data preparation. Following the process of classification in this work as a supervised learning task, all the collected data were labeled as Fall or ADL. Fig. 5 shows a GUI application which is implemented in order to easily trim recorded acceleration signals. The process of reading, trimming and labeling of a sample of fall left side is described in Fig.5. After reading the sample fall data, the start point of recording data was determined manually and its value was saved in the related box as "181". The trimming process was performed by pushing the delete button. After trimming, the start point and the end point of the fall were determined manually and their values were saved in the related boxes as " 230 " and " 334 ". Finally data labeling was performed by considering a binary target signal to show the exact location of a fall and ADL. All these processes are saved by pushing the save button and by pushing the start button the next data is ready to be processed.

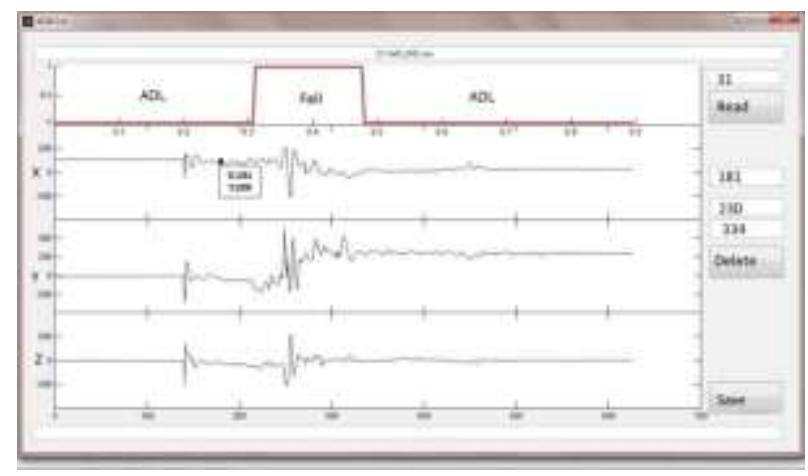

Fig. 5. GUI application for trimming the recorded acceleration signals

\subsection{Windowing technique}

The main classification problem in this work is to distinguish between fall events and normal activities. Since every recorded acceleration signal from different movements has its own pattern, they can be classified 
by a classification algorithm as fall or ADL. Before raw acceleration data going through classification algorithm, they must be preprocessed using a windowing technique in order to increase the classification accuracy. ${ }^{15}$ In this work, windowing technique was used to divide the sensor signal into smaller time segments (i.e., windows) and MLP neural network as a classification algorithm was applied separately on each window, which means each window produces a classification result. In some cases, the windowing technique splits the falling acceleration signals in two separate windows. Some part of a fall signal can exist in one window and the remaining part goes in the next window which reduces the accuracy of classification result significantly. As a solution, sliding window method was applied to cover each fall signal completely at least in one window. In this sliding window method, the signal is divided into windows of fixed length and a fixed overlap size with a previous window. The window size for preprocessing is very important in detection accuracy because a small window will misclassify patterns while a large window has the potential to misclassify a fall and has low performance in fast movement recognition. ${ }^{16}$ Therefore, two different experiments were performed in order to find the best window size. In the first experiment which is a statistical analysis of the recorded acceleration database, the average falling duration time was calculated. Theoretically a perfect window size should cover the whole falling duration to extract maximum related features. As a result of this experiment, the average falling duration was 0.97 seconds with the low variance. Therefore, it is expected to achieve the best result with the window size approximately one second. Other method for evaluating the window size is simply performing a trial and error experiment. In this approach, the features were extracted from different window size from 0.2 seconds to 3 seconds. Then, the proposed neural network was trained with features extracted from each window size. Fig. 6 shows the mean squared error of neural network training performance for different window sizes which lower error means better classification accuracy. Finally, the minimum error was achieved with window size: 1000 (1 second), step-size: 500 and overlap: 500 (0.5 seconds). The overlap was considered in order to include all the falls that may happen between two windows. Fig. 7 shows a sample acceleration signal of fall forward with window-size: 1000, step-size: 500 and overlap: 500 at

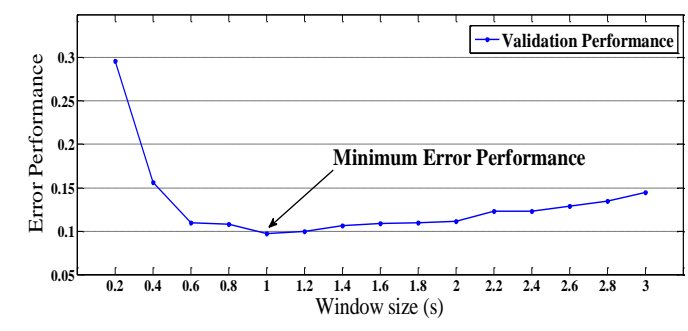

Fig. 6. Error performance as a function of window size

$100 \mathrm{~Hz}$ sample rate. From the Fig. 7, it can be clearly seen that the falling signal is covered completely by just W2. As mentioned before, the falling signal is fragmented by W1 and W3. It is expected that the W2 can detect the fall more accurately.

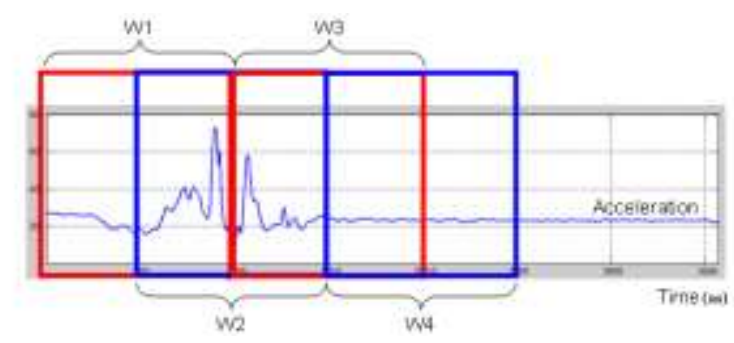

Fig. 7. Sliding window approach

\subsection{Feature extraction}

Following the objective of this work to classify fall patterns from ADL patterns, different features have been extracted from each time window on recorded acceleration data. The complete set of features that were chosen as candidates for this work is composed of two parts: personal information features and acceleration features. Since making a well distributed and balanced database of different activities is the most critical and important part of a classification work, a database of 50 volunteers of men and women with different features such as age, height, weight and sex were collected. This personal information was considered as one part of features that each collected data must have. The most important feature for acceleration signal was extracted from total sum vector. Total Sum vector was determined as in "Eq. (1)":

$$
\bar{A}=\sqrt{\bar{x}^{2}+\bar{y}^{2}+\bar{z}^{2}} .
$$

$\mathrm{X}, \mathrm{Y}$ and $\mathrm{Z}$ represent the accelerometer value in three axes which are in gravity $\mathrm{g}$ range. ${ }^{15}$ Angular 
velocity is another feature that can indicate the volunteers' rotational movement according to each axis. In order to calculate the angular velocity, the direction of acceleration vector (angle) in each axis is required which is calculated as follows ${ }^{17}$ :

$$
\alpha=\cos ^{-1} \frac{\bar{x}}{\bar{A}}, \beta=\cos ^{-1} \frac{\bar{y}}{\bar{A}}, \gamma=\cos ^{-1} \frac{\bar{z}}{\bar{A}} .
$$

According to mentioned equations, angular velocity for each axis is calculated as follows:

$$
\begin{aligned}
& \text { Angular Velocity }_{x}(i)=\alpha(i)-\alpha(i-1) . \\
& \text { Angular Velocity }_{y}(i)=\beta(i)-\beta(i-1) . \\
& \text { Angular Velocity }_{z}(i)=\gamma(i)-\gamma(i-1)
\end{aligned}
$$

Velocity signals which are the integral of acceleration signals were calculated by continuously integrating the acceleration signals in each window. Similarly the position signals which are the integral of velocity signals were calculated by continuously integrating the velocity signals in each window. Features including acceleration, angular velocity, velocity and position signals, different time domain features such as Maximum, Minimum, Mean, Range, Variance and Standard deviation have been extracted as explained in Table 2. By using these features and personal information features, totally 28 features have been extracted.

Table 2. List of extracted features and related time domain features.

\begin{tabular}{llll}
\hline $\begin{array}{l}\text { Acceleration } \\
\text { signal (a) }\end{array}$ & $\begin{array}{l}\text { Velocity signal Position signal (p) } \\
(\mathrm{v})\end{array}$ & $\begin{array}{l}\text { Angular velocity } \\
(\mathrm{av})\end{array}$ \\
\hline $\operatorname{Max} \bar{A}_{a}$ & $\operatorname{Max} \bar{A}_{v}$ & $\operatorname{Max} \bar{A}_{p}$ & $\operatorname{Max} \bar{A}_{a v}$ \\
$\operatorname{Min} \bar{A}_{a}$ & $\operatorname{Min} \bar{A}_{v}$ & $\operatorname{Min} \bar{A}_{p}$ & $\operatorname{Min} \bar{A}_{a v}$ \\
$\operatorname{Mean} \bar{A}_{a}$ & $\operatorname{Mean} \bar{A}_{v}$ & $\operatorname{Mean} \bar{A}_{p}$ & $\operatorname{Mean} \bar{A}_{a v}$ \\
$\operatorname{Range} \bar{A}_{a}$ & $\operatorname{Range} \bar{A}_{v}$ & $\operatorname{Range} \bar{A}_{p}$ & $\operatorname{Range} \bar{A}_{a v}$ \\
$\operatorname{Var} \bar{A}_{a}$ & $\operatorname{Var} \bar{A}_{v}$ & $\operatorname{Var} \bar{A}_{p}$ & $\operatorname{Var} \bar{A}_{a v}$ \\
$\operatorname{Std} \bar{A}_{a}$ & $\operatorname{Std} \bar{A}_{v}$ & $\operatorname{Std} \bar{A}_{p}$ & $\operatorname{Std} \bar{A}_{a v}$
\end{tabular}

\subsection{Proposed MLP neural network}

For complex problems, such as learning to interpret real-world sensor data, Artificial Neural Networks (ANNs) are among the most effective learning methods currently known. They provide a general and practical method for learning real-valued, discrete-valued and vector-valued from linear and non-linear functions. ${ }^{18}$ As explained in our previous work $^{19}$, a multilayer perceptron (MLP) neural network as a commonly used
ANN structure was selected as the most powerful classification algorithm for precise classification of motions and determination of fall or ADL events. It is a three-layer ANN consisting of input layer, hidden layer and output layer. Fig. 8. illustrates the structure of proposed three-layer MLP architecture.

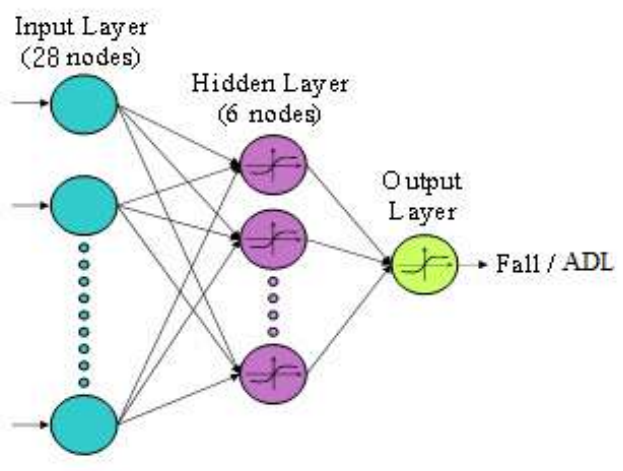

Fig. 8. The structure of proposed MLP

The input layer consists of 28 neurons which represent the number of features that have been extracted. Since fall detection classification is a nonlinear problem, one hidden layer is sufficient for our proposed MLP model. ${ }^{18}$ In order to determine the exact number of neurons in the hidden layer, some trials and errors have been performed. The MLP with different numbers of hidden nodes (1:50) was evaluated. The training process was completed by achieving the minimal error for validation set. Because of the random initialization of the ANNs weights, the result of training will be different for two MLPs with the same structure; therefore, for each number of hidden neurons, the training process was repeated 20 times in order to find the accuracy as a function of hidden neurons by averaging the results. Fig. 9 illustrates that minimum error was achieved with 6 neurons in one hidden layer.

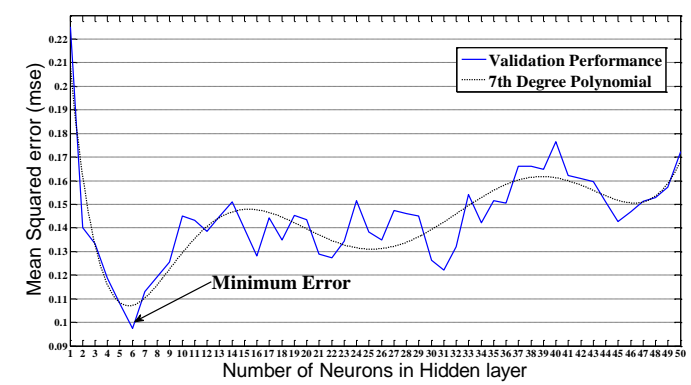

Fig. 9. Mean squared error (mse) as a function of hidden neurons 
Therefore, 6 nodes were considered as hidden nodes. The output layer has one neuron which generates " 1 " as fall and "-1" as ADL.

Randomly, $75 \%$ of total data set (1000 recorded acceleration data) was considered as training data set (750) and 25\% remaining was considered for test set (250). ${ }^{18}$ Backpropagation algorithm was used as the most commonly learning technique for the proposed MLP which uses gradient descent with momentum and adaptive learning rate backpropagation as learning method. The proposed MLP is capable of expressing a rich variety of nonlinear decision surfaces which is appropriate for classification in this work. In order to use the Backpropagation training method, the transfer function must be differentiable. The proposed MLP with different transfer functions such as Tansig, log sigmoid and Pureline were trained for 20 times in order to find the best classification performance and evaluate the consistency of learning. Table 3. shows that minimal error was achieved at 0.0974 using Tansig transfer function. Its standard deviation shows that in several training processes, nearly similar results were achieved. The log sigmoid could not classify the fall from ADL patterns because this transfer function can only generate positive values between 0 to +1 while the ADL are shown with -1 in training vectors. Although the Pureline transfer function generates all positive and negative values, it could not achieve better result compared with Tansig function. Finally, the minimum error for the proposed neural network was achieved at 0.0974 after 170 epochs with Tansig as transfer function and 6 neurons in one hidden layer.

Table 3. Performance of trained MLP with different transfer functions.

\begin{tabular}{llll}
\hline Transfer function & $\begin{array}{l}\text { Average } \\
\text { performance }\end{array}$ & Std performance & $\begin{array}{l}\text { Minimal error } \\
\text { performance }\end{array}$ \\
\hline Pureline & 0.1489 & $7.39 * 10^{-5}$ & 0.1486 \\
Tansig & 0.1165 & 0.0101 & 0.0974 \\
Logsig & 0.7743 & 0.0147 & 0.7467
\end{tabular}

\section{Results and Discussion}

Fall detection result is considered to be positive $(+1)$ if the classifier properly recognizes a fall and to be negative (-1) if it does not. Therefore, there are four $\operatorname{cases}^{20}$ :

(i) True positive (TP): A fall occurs, the device detects it. (ii) False positive (FP): The device announces a fall, but it did not occur.

(iii) True negative (TN): ADL movement is performed, the device does not declare a fall.

(iv) False negative (FN): A fall occurs but the device does not detect it

The false positive value shows the number of falling alarms that the system generates without having any real fall therefore; it will disturb the users by giving false alarms if this parameter increases. On the other hand, false negative value shows the number of falls which were not recognized as fall by the system which is so fatal for the elderly. The main objective of this work is to decrease both false positive and false negative simultaneously. True positive, False positive (type I error), True negative and False negative (type II error) were calculated using the information of Table 4. In order to evaluate the response to these 4 cases, 2 criteria are proposed:

- Sensitivity: It is the capacity to detect a fall.

$$
\text { Sensitivity }=T P /(T P+F N) .
$$

It is used to evaluate the capacity to detect a fall. Higher sensitivity indicates that the fall detection system could detect falls accurately with less false alarms.

- Specificity: It is the capacity to detect only a fall.

$$
\text { Specificity }=T N /(T N+F P) .
$$

It is used to evaluate the capacity to avoid false positives. Intuitively, it is the capacity to detect a fall only if it really occurs.

In addition to sensitivity and specificity, other criteria such as accuracy, positive predictive value and negative predictive value were used to evaluate the fall. All these criteria are depicted in the following sentences:

- Accuracy: It is the ability to distinguish and detect both fall (TP) and ADL movement (TN)

$$
\text { Accuracy }=(T P+T N) /(P+N) .
$$

Where $\mathrm{P}$ and $\mathrm{N}$ are, respectively, the number of falls is performed and the number of ADL is performed.

- Positive predictive value: It tells the probability that we actually have a fall if the device detects the fall.

Positive predictive value $=T P /(T P+F P)$. (9)

- Negative predictive value: It tells the probability that we actually do not have the fall if the device detects the fall.

Negative predictive value $=T N /(T N+F N)$.

According to these Formulas, the experimental results of fall detection were calculated and shown in Table 4. 
Table 4. Fall detection statistical result.

\begin{tabular}{|c|c|c|c|c|}
\hline Total $=250$ & Real & & & \\
\hline \multirow[t]{3}{*}{ Test } & Positive $=138$ & True positive $=127$ & False positive $=10$ & Positive predictive value $=92.7 \%$ \\
\hline & Negative $=112$ & False negative $=11$ & True negative $=102$ & Negative predictive value $=90.26 \%$ \\
\hline & & Sensitivity $=92.03 \%$ & Specificity $=91.07 \%$ & Accuracy $=91.6 \%$ \\
\hline
\end{tabular}

As a result of this work, falls can be distinguished from normal activities with a sensitivity of $92.03 \%$, specificity of $91.07 \%$ and accuracy of $91.6 \%$ from a total of 250 test data.

\section{Conclusion}

In this paper a waist worn fall detection system has been proposed. The acceleration signals were measured using ADXL345 accelerometer sensor from volunteers' falls and ADL movements. MLP neural network was applied as learning and classification algorithm to classify falling patterns from ADL. It was found that the minimum error achieved by 6 neurons in one hidden layer after 170 epochs with tansig transfer function. The fall detection result showed that falls can be distinguished from ADL with a sensitivity of $92.03 \%$, specificity of $91.07 \%$ and accuracy of $91.6 \%$ from a total of 250 test data. It has the potential to perform even better classification for acceleration data with future customized development and fine tuning of its parameters. The trained MLP will be used for further development in order to make a pervasive device that can be accessible for elderly real time fall detection in every situation.

\section{Acknowledgements}

The authors would like to thank the students and staffs of department of computer and communication system engineering, University Putra Malaysia and all other volunteers for their kind cooperation with collecting the experimental data for this project.

\section{References}

1. M.E. Tinetti and C.S. Williams, "Falls, injuries due to falls, and the risk of admission to a nursing home," New England Journal of Medicine, vol. 337, no. 18, pp. 127984, 1997.

2. Bourke, Alan K.; van de Ven, Pepijn W.J.; Chaya, Amy E.; OLaighin, Gearoid M.; Nelson, John; , "The design and development of a long term fall detection system incorporated into a custom vest for the elderly.," Engineering in Medicine and Biology Society, 2008. EMBS 2008. 30th Annual International Conference of the IEEE, pp.2836-2839, 20-25 Aug 2008.

3. Xinguo $\mathrm{Yu}$; , "Approaches and principles of fall detection for elderly and patient," e-health Networking, Applications and Services, 2008. HealthCom 2008. 10th International Conference on , pp.42-47, 7-9 July 2008

4. Hijaz, F.; Afzal, N.; Ahmad, T.; Hasan, O.; , "Survey of fall detection and daily activity monitoring techniques," Information and Emerging Technologies (ICIET), 2010 International Conference on , pp.1-6, 14-16 June 2010

5. Kangas, M.; Konttila, A.; Winblad, I.; Jamsa, T.; , "Determination of simple thresholds for accelerometrybased parameters for fall detection," Engineering in Medicine and Biology Society, 2007. EMBS 2007. 29th Annual International Conference of the IEEE, pp.13671370, 22-26 Aug. 2007

6. Bourke A.K, O’Brien J.V, Lyons G.M., "Evaluation of a threshold based triaxial accelerometer fall detection algorithm". Gait Posture 2007, vol. 26, no. 2, pp. 194 199.

7. Jiewen Zheng; Guang Zhang; Taihu Wu; , "Design of Automatic Fall Detector for Elderly Based on Triaxial Accelerometer," Bioinformatics and Biomedical Engineering , 2009. ICBBE 2009. 3rd International Conference on , pp.1-4, 11-13 June 2009

8. Zhang $\mathrm{T}$, Wang $\mathrm{J}, \mathrm{Xu} \mathrm{L}$ and Liu $\mathrm{P}$, Using wearable sensor and NMF algorithm to realize ambulatory fall detection. Advances in Natural Computation: Part 2 (Lecture Notes in Computer Science vol 4222) (Berlin: Springer) pp 488-91.

9. Zhang T, Wang J, Xu L and Liu P; , "Fall detection by wearable sensor and one-class SVM algorithm," Engineering Intelligent Computing in Signal Processing and Pattern Recognition, Lecture Notes in Control and Information Sciences, Volume 345, pp.858-863,2006

10. ADXL345: http://www.analog.com/

11. ADXL345 Evaluation Board: http://www.sparkfun.com/

12. Qiang Li; Stankovic, J.A.; Hanson, M.A.; Barth, A.T.; Lach, J.; Gang Zhou; , "Accurate, Fast Fall Detection Using Gyroscopes and Accelerometer-Derived Posture Information," Wearable and Implantable Body Sensor Networks, 2009. BSN 2009. Sixth International Workshop on, pp.138-143, 3-5 June 2009 
13. Hwang, J.Y.; Kang, J.M.; Jang, Y.W.; Kim, H.C.; , "Development of novel algorithm and real-time monitoring ambulatory system using Bluetooth module for fall detection in the elderly," Engineering in Medicine and Biology Society, 2004. IEMBS '04. 26th Annual International Conference of the IEEE , pp.2204-2207, 1-5 Sept. 2004

14. M. Torrent.; A. Bourke.; X. Parra.; A. Català.; "Ambulatory Mobility Characterization Using Body Inertial Systems: An Application to Fall Detection", in Proc. IWANN (1), 2009, pp.1129-1136.

15. S. Preece et al., "Activity identification using bodymounted sensors - a review of classification techniques," Physiological Measurement, vol. 30, no. 4, pp. R1-R33, April 2009.

16. Estudillo-Valderrama, M.A.; Roa, L.M.; Reina-Tosina, J.; Naranjo-Hernandez, D.; , "Design and Implementation of a Distributed Fall Detection System-Personal Server," Information Technology in Biomedicine, IEEE Transactions on , vol.13, no.6, pp.874-881, Nov. 2009

17. Hsu-Yang Kung; Chin-Yu Ou; Shin-Di Li; Chun-Hao Lin; Hong-Jie Chen; Yu-Lun Hsu; Miao-Han Chang; Che-I Wu; , "Efficient movement detection for human actions using triaxial accelerometer," Consumer Electronics (ICCE), 2010 Digest of Technical Papers International Conference on , pp.113-114, 9-13 Jan. 2010

18. Tom M. Mitchell. Machine learning. USA: The McGrawHill Science, 1997, pp. 81-126.

19. Hamideh Kerdegari.; Khairulmizam Samsudin.; Abd Rahman Ramli.; Saeid Mokaram.; , " Evaluation of Fall Detection Classification Approaches," presented at the International Conference on Intelligent and Advanced Systems (ICIAS), Malaysia, 12-14 June 2012

20. Noury, N.; Fleury, A.; Rumeau, P.; Bourke, A.K.; Laighin, G.O.; Rialle, V.; Lundy, J.E.; , "Fall detection Principles and Methods," Engineering in Medicine and Biology Society, 2007. EMBS 2007. 29th Annual International Conference of the IEEE , pp.1663-1666, 2226 Aug. 2007 